\title{
ABDOMINAL ULTRASOUND DIAGNOSTICS AT COVID-19
}

\author{
RENATA DOS SANTOS, JAMILI EL BACHA, LEONARDO PIBER
}

\begin{abstract}
COVID-19 affects multiple systems, manifesting itself in the most diverse clinical or associated forms. The world medical community is still learning about this entity and a pandemic as a whole. The literature has publications that formalize the abdominal manifestations of COVID-19, as well as its most adequate diagnostic methods. Ultrasonography stands out as a method of diagnosis and auxiliary procedures in therapeutics.

The purpose of this is to review and study abdominal ultrasound findings in patients with COVID-19. This is a narrative literature review, searching the Pubmed, Scielo and LILACS database, using the following descriptors: ultrasonography, COVID-19 and abdomen. All articles with ultrasound images published since December 2019 were included. Abdominal ultrasound images of cases diagnosed with COVID-19 were included.

A B-mode analysis, associated with Doppler, is associated with the vascular involvement characteristic of this viral entity. Among the recent publications on the subject, changes related to portal venous gas due to mesenteric vascular injury, portal vein thrombosis, distended gallbladder, biliary stasis, diffusely bulky pancreas without focal lesions or gallstones, areas of renal infarction, are evidenced. ascites, thickening of the intestinal wall, interstitial and / or hemorrhagic cystitis.
\end{abstract}

The mastery of ultrasound findings related to COVID-19 abdominal changes, if necessary, as an urgent contemporary need.

\section{KEYWORDS: ULTRASONOGRAPHY, IMAGING DIAGNOSIS, ABDOMEN, COVID-19, CORONAVIRUS}

\section{INTRODUCTION}

Coronavirus 2019 disease (COVID-19), caused by the severe acute respiratory syndrome coronavirus 2 (SARSCoV-2), was officially reported in December 2019 in Wuhan City, Hubei Province, China, and has since led to a pandemic.1 Disease severity ranges from subclinical infections to serious illnesses that require hospital admission. As the number of infected individuals increases, it is noticed that not only the lungs but also other organs can be affected by the virus.

Gastrointestinal symptoms, such as diarrhea, vomiting, nausea or abdominal pain, are frequent in patients with COVID-19. Additionally, alimentary tract symptoms may precede the respiratory presentation of SARS-CoV-2 infection. This can lead to late diagnosis and inadequate management of infected patients. ${ }^{2}$

Viral RNA was found in faecal samples, despite negative breath tests and in the gastrointestinal epithelium, suggesting a possible route of faecal-oral transmission. ${ }^{3}$

SARS-CoV-2 is thought to gain access to cells through surface expression of the angiotensin- 2 converting enzyme (ACE2). Thus, it is assumed that tissues with high levels of ACE2 expression are susceptible to direct infection. ACE2 surface expression is most abundant in lung alveolar epithelial cells, small bowel enterocytes, and vascular endothelium. The large amount of surface expression of ACE2 in the gastrointestinal tract and even less in the biliary epithelium have been offered as possible explanations for gastrointestinal symptoms and liver damage. In addition, SARS-CoV-2 has been identified in stool samples from a substantial proportion of infected patients. ${ }^{4}$

Thus, in this severe scenario of an acute pandemic, patients who arrive at the emergency room with an acute abdomen should also be considered for COVID-19 infection, even in the absence of respiratory symptoms. ${ }^{5}$

In the clinical investigation of this patient, imaging exams are common in surgical decision-making and can help to understand the abdominal phenomena in SARS-CoV-2 infection. Therefore, it is important that ultrasonographers are aware of abdominal imaging findings in patients with COVID-19.

Considering the cost-effectiveness of the imaging used, ultrasound is a non-ionizing, portable, bedside imaging modality with a high diagnostic impact in emergencies and intensive care units in pandemics. In addition, ultrasound can provide
1. Universidade Santo Amaro

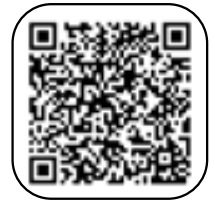

\section{MAILING ADDRESS}

LEONARDO DE SOUZA PIBER

Endereço: Rua Marechal Deodoro, 135 apto 62B, Santo Amaro,

São Paulo, SP, Brasil, CEP 04738-000

E-mail: leonardopiber@gmail.com 
real-time diagnostic and therapeutic interventions. ${ }^{6}$

Contrast ultrasonography can also play an important role, as it offers the possibility of analyzing dynamic microcirculatory disorders in real time in a dynamic way, without risk to the kidneys and thyroid gland, even in severe cases of progressive disease.?

The aim of this study is to review and study abdominal ultrasound findings in patients with COVID-19.

\section{METHODS}

Narrative literature review, searching the Pubmed, Scielo and Lilacs databases, using the following descriptors: "ultrasonography, COVID-19 and abdomen" (ultrasonography AND COVID-19 AND abdomen). All articles with ultrasound images, published since December 2019, in Portuguese and English, were included; and those articles that do not continue with images or that were not in accordance with the objective of this research were excluded.

\section{RESULTS AND DISCUSSION}

The liver is frequently affected by COVID-19. The ACE2 receptor has been found to be expressed in the liver, specifically in cholangiocytes (bile duct epithelial cells) rather than in hepatocytes, thus viral infection with SARS-CoV-2 alters the barrier and bile acid transport functions of the cholangiocytes through dysregulation of genes involved in the formation of closed junctions and the transport of bile acid. This could explain the accumulation of bile acids and resulting liver damage. ${ }^{8}$

Figure 1 shows echogenic ramifications, with acoustic reverberation, corresponding to portal venous gas that may be related to intestinal pneumatosis due to ischemia or infarction of the intestinal segment, in a 34-year-old patient with abnormal liver enzymes.

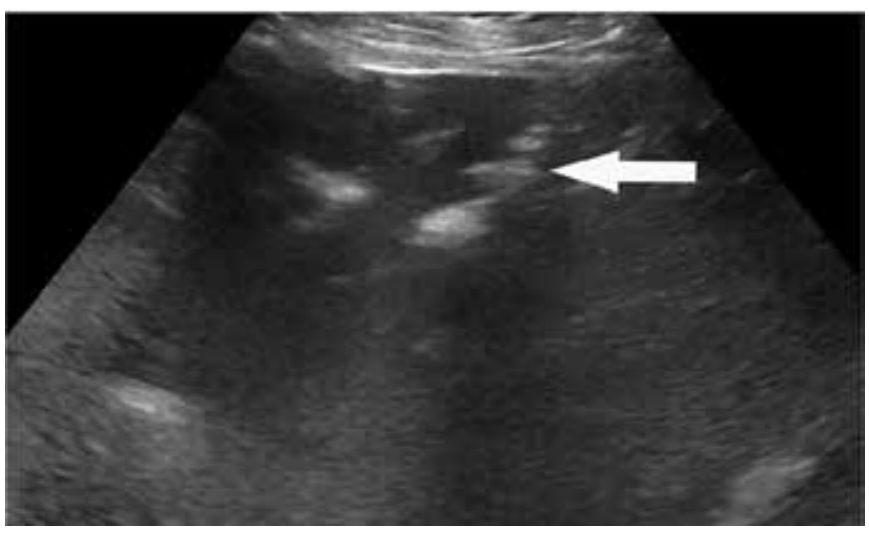

Figure 1 - Liver ultrasound showing echogenic ramifications in peripheral portal segments. ${ }^{4}$

Thrombosis of mesenteric, splenic and portal vein vessels may be present in COVID-19 infection, even in non-severe cases of the disease. ${ }^{9}$

Figure 2 refers to the importance of Doppler analysis of the portal vein. This is a 44-year-old man with a recent history of COVID-19 disease, who presented severe abdominopelvic pain of progressive and insidious onset. B-mode thrombus is not characterized; however, abnormal flow is observed on Doppler, with a diagnosis of thrombosis of the portal vein.9 Portal vein with signs of cavernomatous transformation on Doppler study.

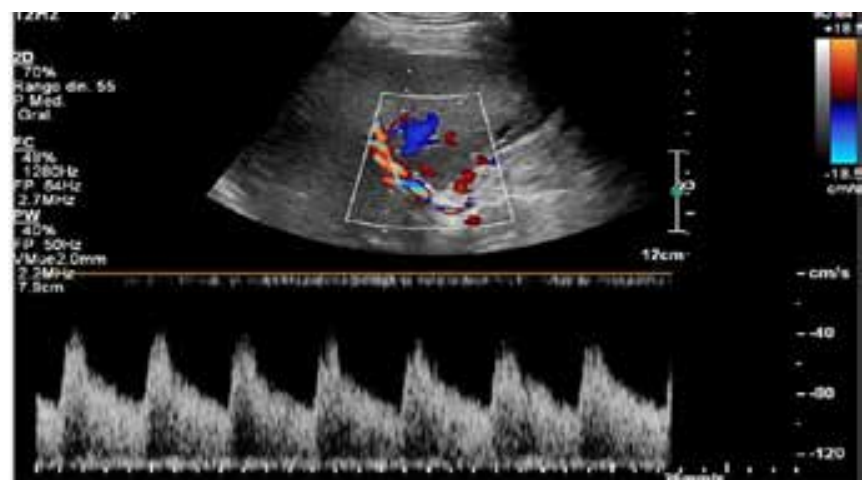

Figure 2 - Ultrasonography with Doppler analysis, whose diagnosis of thrombosis was evidenced by the change in flow. The thrombus was not characterized in B-mode. ${ }^{9}$

Biliary stasis can be diagnosed by gallbladder recognition and intrahepatic biliary ductal dilatation, without a causal obstructive mass or calculus. It is important to note that acute cholecystitis can develop as a result of biliary stasis. ${ }^{8}$

Figures $3 \mathrm{~A}$ and $3 \mathrm{~B}$ show a gallbladder with increased volume, thick bile, thickened wall and hyperflow on Doppler. These findings were related to the diagnosis of acute cholecystitis in a 64-year-old man with sepsis. He underwent cholecystostomy and bacterial bile culture was negative, confirming the diagnosis of biliary stasis. ${ }^{8}$

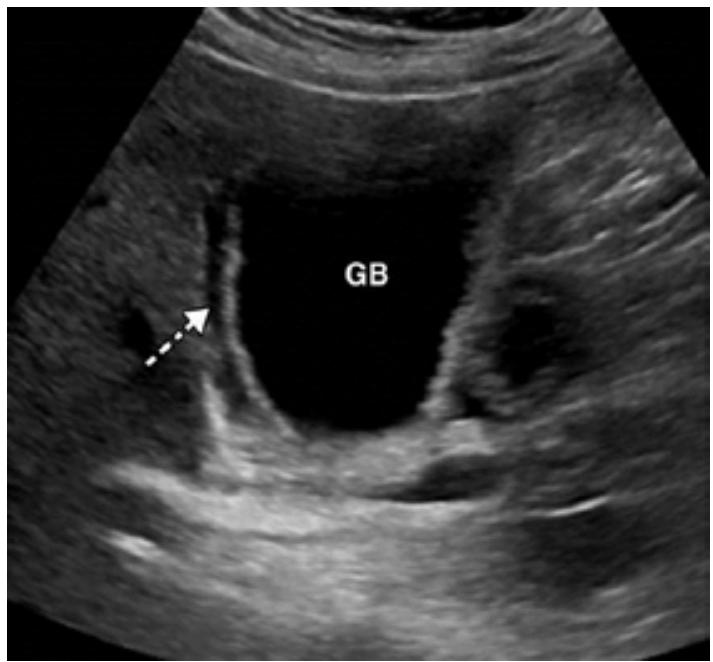

Figure 3A - Gallbladder ultrasonography (B mode) shows gallbladder with increased volume (GB) and significant wall edema (white dashed arrow). Findings suggest biliary stasis and acute cholecystitis. ${ }^{8}$ 


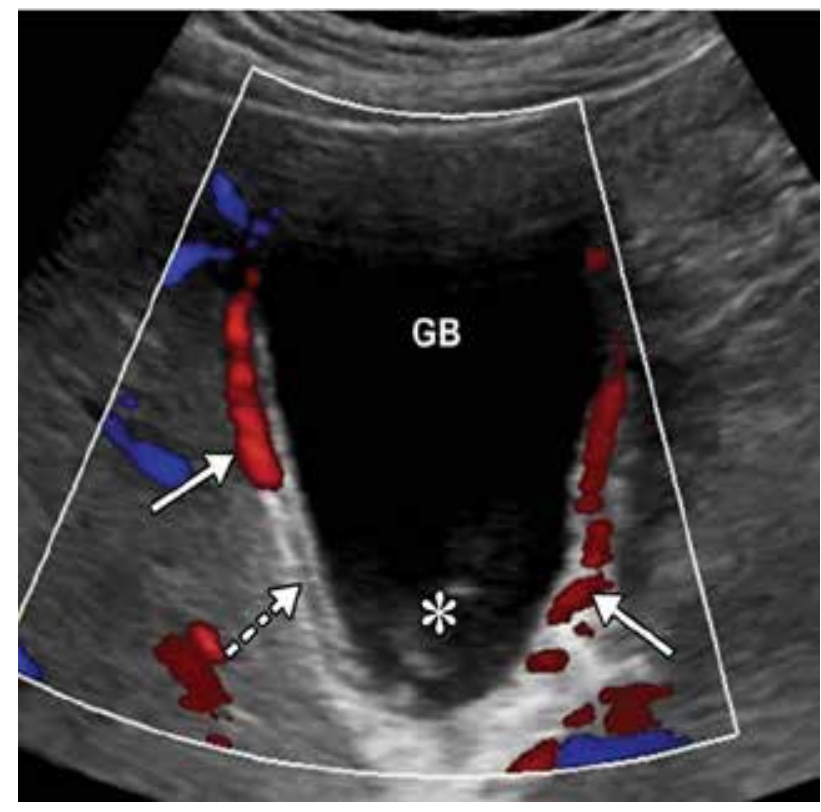

Figure 3B - Gallbladder ultrasonography (color Doppler) showing gallbladder with increased volume (GB) and thick bile $\left({ }^{*}\right)$. Significant wall edema (white dashed arrow) and hyperflow (solid white arrows). Findings suggest biliary stasis and acute cholecystitis. ${ }^{8}$

Figures $4 \mathrm{~A}$ and $4 \mathrm{~B}$ show a case similar to the previous one, this is an 83-year-old man with elevated liver enzymes and sepsis, with a distended gallbladder and thick bile. These findings are suggestive of cholestasis. The patient also underwent ultrasound-guided cholecystostomy. The bile was not infected. ${ }^{4}$

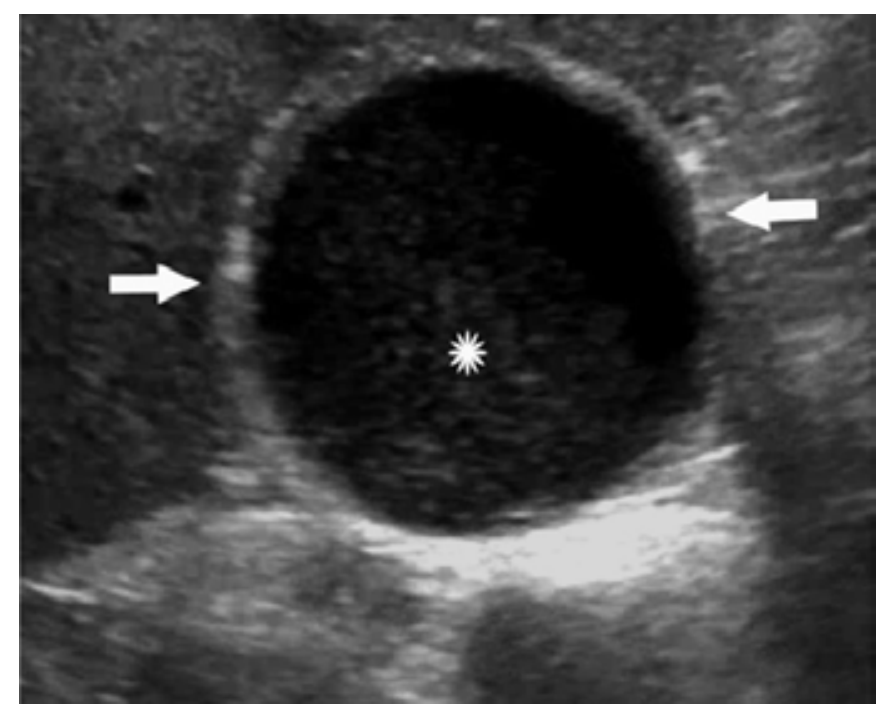

Figure 4A - Gallbladder ultrasonography (cross section) showing distension (arrows) and containing thick bile $\left({ }^{*}\right)$, suggestive of cholestasis. ${ }^{4}$

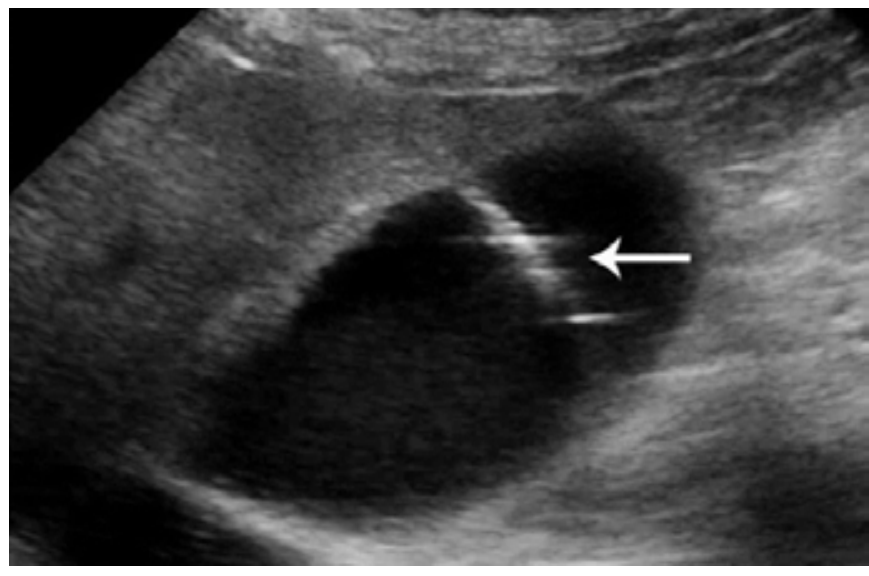

Figure 4B - Gallbladder ultrasonography (Iongitudinal section) showing a needle during the guided cholecystostomy procedure. ${ }^{4}$

Figure 5 depicts a 4-year-old boy with a medical history of prematurity and mild asthma who presented with fever, abdominal pain, diarrhea, hypotensive shock, and evidence of myocardial dysfunction with positive coronavirus disease. Image shows gallbladder wall thickening but no gallstones. ${ }^{10}$

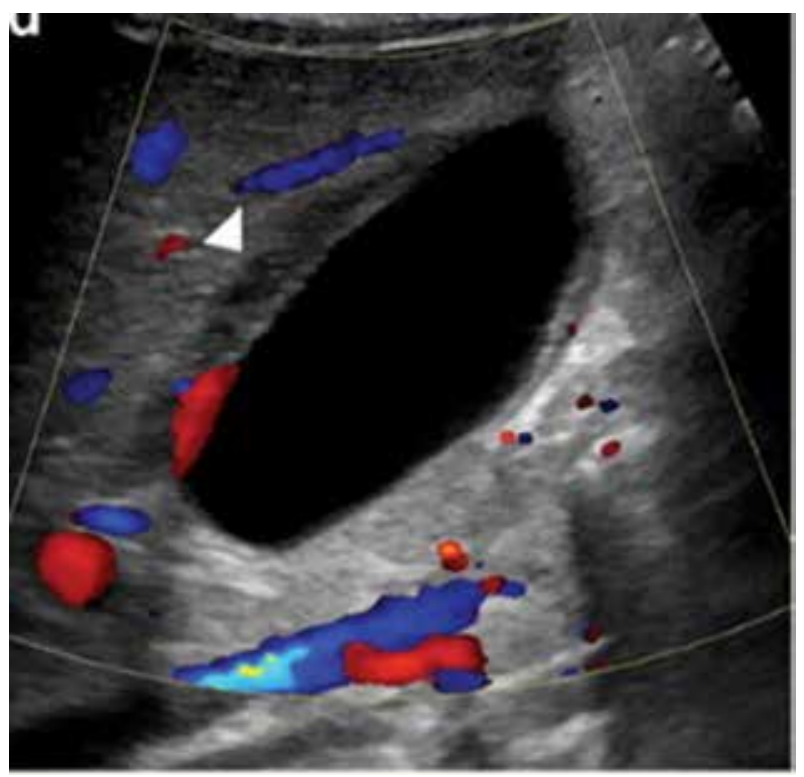

Figure 5 - Gallbladder ultrasonography (longitudinal section) with Doppler analysis, showing gallbladder wall thickening (arrowhead). ${ }^{10}$

Solid organ infarctions can also be visualized on abdominal imaging in patients with COVID-19, affecting the kidney and liver.

Figure 6 refers to a 52-year-old patient, severely affected 
by COVID-19, evolving to orotracheal intubation, presenting oliguria, with high creatinine levels, whose diagnosis was renal infarction.

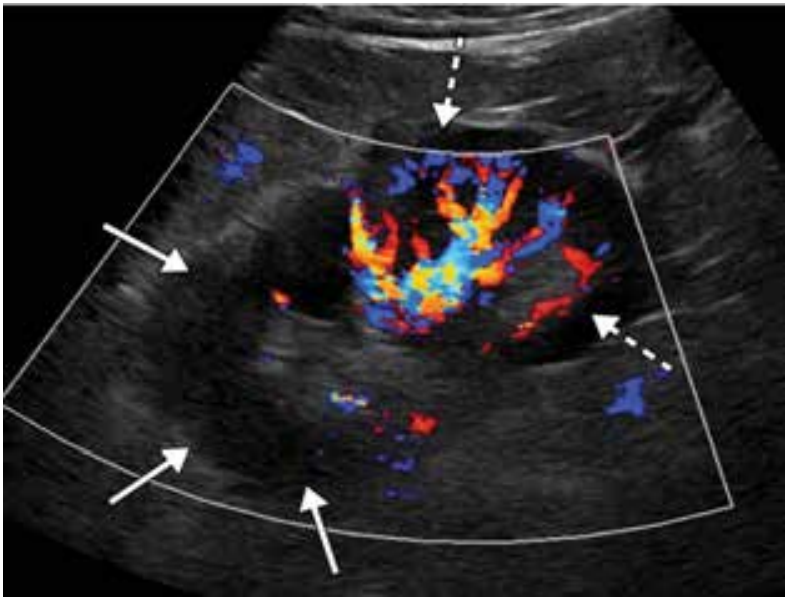

Figure 6 - Renal ultrasound (longitudinal section) with Doppler analysis showing a focal area of hypoperfusion (solid arrows) in the upper pole of the left kidney, compatible with infarction. Normal perfusion (dashed arrows) is seen at the lower pole of the kidney. ${ }^{8}$

Pancreatic lesion was described in COVID-19 and is believed to be the result of direct or indirect mechanisms. The direct mechanism stems from a cytopathic effect mediated by local viral replication (pancreatic islet and exocrine gland cells have abundant ACE2 receptors), while the indirect mechanism refers to a systemic response to respiratory failure or a self-induced harmful immune virus response. Up to $17 \%$ of patients with COVID-19 have pancreatic damage. 8

Figure 7 shows an examination of a 47 -year-old female patient with diffusely enlarged pancreas suggestive of acute pancreatitis, without focal changes or gallstones.

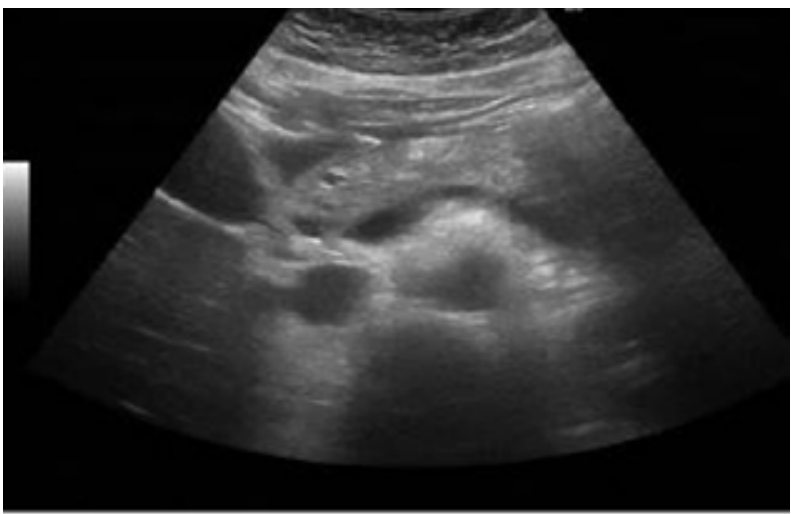

Figure 7 - Pancreas ultrasonography showing increased volume. ${ }^{3}$
Figure 8 shows an examination of a seven-year-old girl with diffuse pancreas enlargement (black arrows) and heterogeneous pancreatic echogenicity (white arrow); findings related to acute pancreatitis. ${ }^{11}$

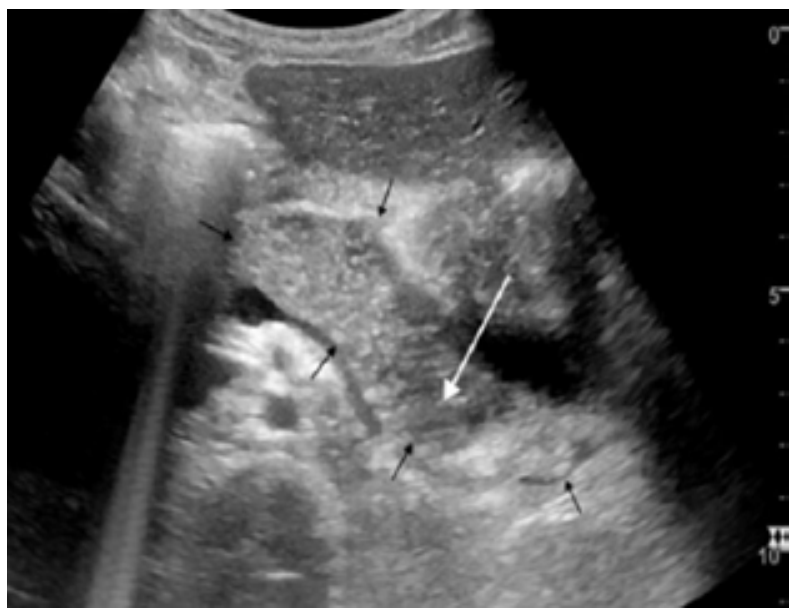

Figure 8 - Pancreas ultrasonography (black arrows) reveals diffuse pancreas enlargement and heterogeneous pancreatic echogenicity (white arrow). ${ }^{11}$

Intestinal involvement results from direct viral infection, virus-induced intestinal inflammation, or intestinal wall ischemia. In patients with COVID-19, bowel wall ischemia occurs in the context of macro or micro arterial thrombosis or venous occlusion, with congestion and mesenteric inflammation. ${ }^{8}$

Figures 9A and 9B are of a seven-year-old girl who presented with fever, abdominal pain, diarrhea, rash, and evidence of myocardial dysfunction with a diagnosis of COVID-19. This child was initially diagnosed with appendicitis. However, she evolved with small-volume ascites and mesenteric edema adjacent to an enlarged borderline appendix and thickened distal ileum segment. ${ }^{10}$

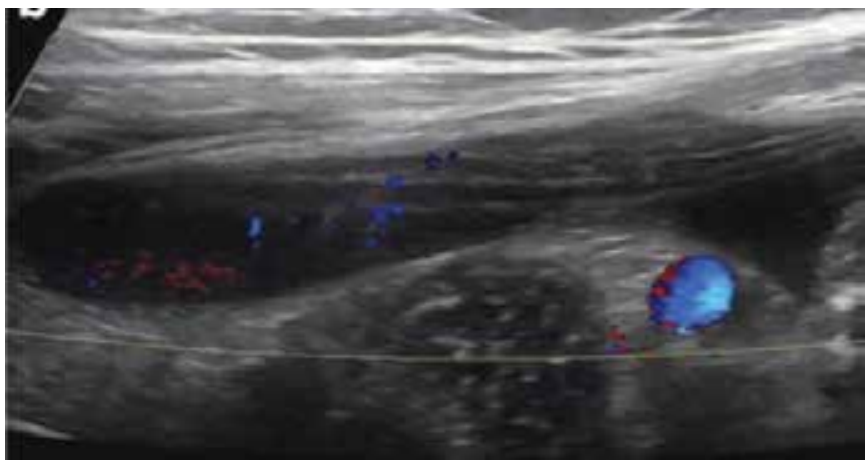

Figures 9A - Abdominal ultrasound with Doppler analysis of the right lower abdominal quadrant demonstrates small volume ascites. ${ }^{10}$ 


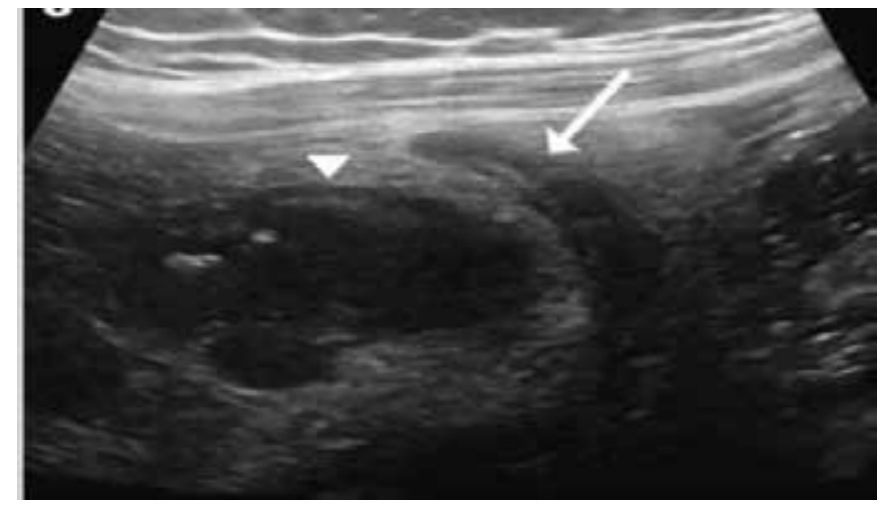

Figures 9B - B-mode abdominal ultrasound of the right lower abdominal quadrant demonstrates mesenteric edema adjacent to an enlarged borderline appendix (white arrow) and a thickened loop of distal ileum (white arrowhead). ${ }^{10}$

Figure 10 shows the right lower abdominal quadrant of a 15-year-old girl, who presents with fever, abdominal pain, conjunctivitis, headache and hypotensive shock. Image shows thickening of the intestinal wall of the distal ileum with surrounding mesenteric edema. ${ }^{10}$

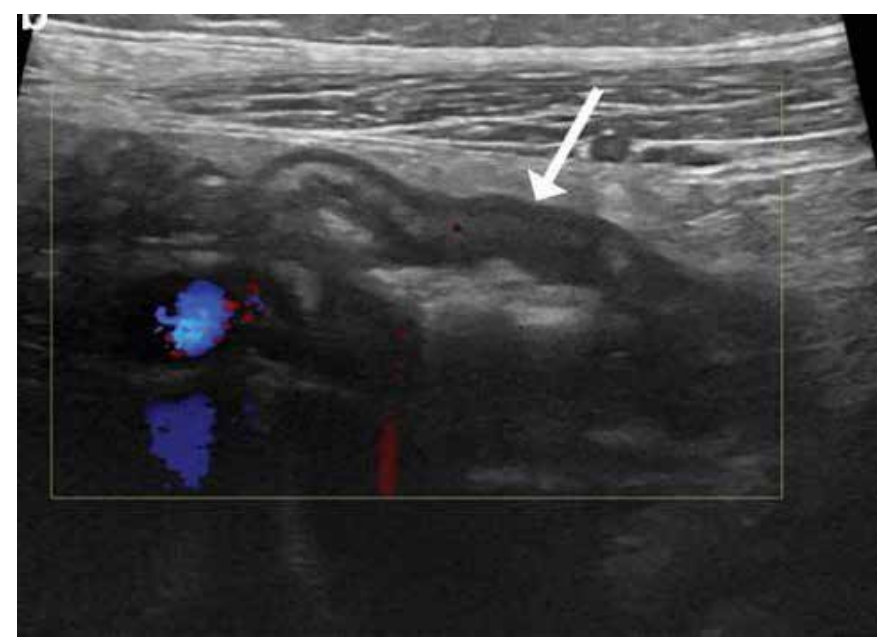

Figure 10 - Abdominal ultrasound with Doppler analysis of the right lower abdominal quadrant shows thickening of the intestinal wall of the distal ileum (arrow) with surrounding mesenteric edema. ${ }^{10}$

The sonographic findings in Figure 11 may correspond to interstitial cystitis and/or hemorrhagic cystitis in an 85-yearold male patient with pneumonia caused by COVID-19, who progressed to hematuria and acute renal failure. ${ }^{8}$

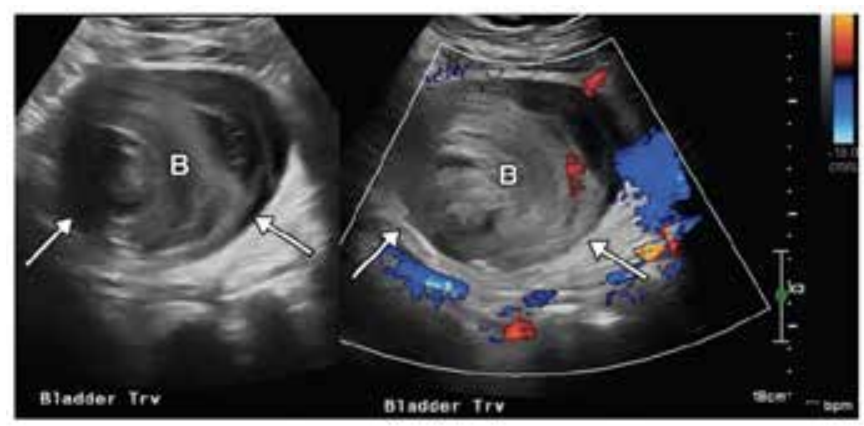

Figure 11 - B-mode bladder ultrasound and Doppler analysis, respectively, showing a distended urinary bladder (B) filled with avascular heterogeneous echogenic material, indicative of vesicle bleed (arrows). ${ }^{8}$

\section{CONCLUSION}

Ultrasonography is an important method in the abdominal assessment of patients diagnosed with COVID-19. B-mode analysis, associated with Doppler, is indicated due to the characteristic vascular involvement of this viral entity. Among recent publications on the subject, changes related to portal venous gas due to mesenteric vascular injury, portal vein thrombosis, distended gallbladder, biliary stasis, diffusely voluminous pancreas without focal lesions or gallstones, areas of renal infarction, ascites, intestinal wall thickening, interstitial and/or hemorrhagic cystitis.

The domain of ultrasound findings related to abdominal changes in COVID-19 has established itself as an urgent contemporary need.

\section{REFERENCES}

1. Adapa S, Aeddula NR, Konala VM, Chenna A, Naramala S, Madhira $\mathrm{BR}$, et al. COVID-19 and renal failure: challenges in the delivery of renal replacement therapy. J Clin Med Res 2020;12(5): 276-285.

2. Smyk W, Janik MK, Portincasa P, Milkiewicz P, Lammert F, Krawczyk M. COVID-19: Focus on the lungs but do not forget the gastrointestinal tract. Eur I Clin Invest. 2020;50(9): e 13276.

3. Hadi A, Werge M, Kristiansen KT, Pedersen UG, Karstensen JG, Novovic S, et al. Coronavirus Disease-19 (COVID-19) associated with severe acute pancreatitis: Case report on three family members Pancreatology 2020;20(4): 665-667.

4. Bhayana R, Som A, Li MD, Carey DE, Anderson MA, Blake MA, et al. Abdominal Imaging findings in COVID-19: Preliminary observations. Radiology. 2020;297(1): 201908.

5. Lima DS, Ribeiro MAF, Gallo G, Di Saverio S. Role of chest CT in patients with acute abdomen during the COVID-19 era. Br J Surg. 2020;107(7): e196.

6. Galluccio F, Ergonenc T, Yamak Altinpulluk E, Perez Herrero M, De la Torre Carpente MDM, Capassoni M, et al. Role of point of care ultrasound in COVID-19 pandemic: what lies beyond the horizon? Med Ultrason. 2020;22(4): 461-468.

7. Jung EMM, Stroszczynski C, Jung F. Contrast enhanced ultrasonography (CEUS) to detect abdominal microcirculatory disorders in severe cases of COVID-19 infection: First experience. Clin Hemorheol Microcirc. 2020;74(4): 353-361.

8. Revzin M V, Raza S, Srivastava NC, Warshawsky R, D'Agostino C, Malhotra A, et al. Multisystem imaging manifestations of COVID-19, Part 2: From cardiac complications to pediatric manifestations. Radiographics. 2020;40(7): 1866-1892.

9. Alemán W, Cevallos LC. Subacute mesenteric venous thrombosis secondary to COVID-19: A late thrombotic complication in a non 
severe patient. Radiol Case Reports. 2021;16(4): 899-902.

10. Fenlon III EP, Chen S, Ruzal-Shapiro CB, Jaramillo D, Maddocks ABR. Extracardiac imaging findings in COVID-19-associated multisystem inflammatory syndrome in children. Pediatr Radiol. 2021;12:19.

11. Alloway BC, Yaeger SK, Mazzaccaro RJ, Villalobos T, Hardy SG. Suspected case of COVID-19-associated pancreatitis in a child. Radiol Case Reports. 2020;15 